\title{
Gender identification from high-pass filtered vowel segments: The use of high-frequency energy
}

\author{
Jeremy J. Donai ${ }^{1}$ • Norman J. Lass ${ }^{1}$
}

Published online: 17 June 2015

(C) The Psychonomic Society, Inc. 2015

\begin{abstract}
The purpose of this study was to examine the use of high-frequency information for making gender identity judgments from high-pass filtered vowel segments produced by adult speakers. Specifically, the effect of removing lowerfrequency spectral detail (i.e., F3 and below) from vowel segments via high-pass filtering was evaluated. Thirty listeners (ages 18-35) with normal hearing participated in the experiment. A within-subjects design was used to measure gender identification for six 250-ms vowel segments (/æ/, /I /, /3 /, / / /, / $\mathrm{o} /$, and $/ \mathrm{u} /$ ), produced by ten male and ten female speakers. The results of this experiment demonstrated that despite the removal of low-frequency spectral detail, the listeners were accurate in identifying speaker gender from the vowel segments, and did so with performance significantly above chance. The removal of low-frequency spectral detail reduced gender identification by approximately $16 \%$ relative to unfiltered vowel segments. Classification results using linear discriminant function analyses followed the perceptual data, using spectral and temporal representations derived from the high-pass filtered segments. Cumulatively, these findings indicate that normalhearing listeners are able to make accurate perceptual judgments regarding speaker gender from vowel segments with low-frequency spectral detail removed via high-pass filtering. Therefore, it is reasonable to suggest the presence of perceptual cues related to gender identity in the high-frequency region of naturally produced vowel signals. Implications of these findings and possible mechanisms for performing the
\end{abstract}

Jeremy J. Donai

jeremy.donai@mail.wvu.edu

1 Department of Communication Sciences and Disorders, West Virginia University, P.O. Box 6122, Morgantown, WV 26506, USA gender identification task from high-pass filtered stimuli are discussed.

Keywords Bandwidth $\cdot$ Gender $\cdot$ High-frequency energy . Identification $\cdot$ Vowels

The ability of normal-hearing listeners to identify speaker gender from an audio signal has been found to be dependent on acoustic information, including the fundamental frequency (F0) and the low-frequency vocal tract resonance characteristics (i.e., formants). Specifically, research has shown listeners to be exceptionally accurate when making this perceptual judgment using information from the low-frequency region (i.e., below F3) of the speech spectrum (e.g., Gelfer \& Bennett, 2013; Gelfer \& Mikos, 2005; Lass, Hughes, Bowyer, Waters, \& Bourne, 1976; Poon \& Ng, 2011; Skuk \& Schweinberger, 2014; Whiteside, 1998). Consequently, substantial research examining the perceptual nature of this spectral region and its contribution to gender identity judgments has been undertaken (see the references above). Conversely, there is a paucity of research examining the use of high-frequency acoustic cues for gender determination. This study reports gender identification results for normalhearing listeners using high-frequency acoustic information from naturally produced, high-pass filtered vowel segments.

Early research by Lass et al. (1976) evaluated the ability of normal-hearing listeners to determine speaker gender from naturally produced vowel segments. The listeners in this experiment were presented 500-ms vowel segments from ten male and ten female speakers in three conditions: (1) unfiltered, (2) low-pass filtered at $255 \mathrm{~Hz}$, and (3) whispered. Although F0 was found to be a salient acoustic cue involved in identifying speaker gender, listeners still performed above chance $(75 \%)$ on the gender identification task in the 
condition lacking any F0 information (i.e., whispered speech). Therefore, information from vocal-tract resonance properties (formants) also provides cues to gender identity.

Whiteside (1998) examined gender identification abilities for ten synthesized vowels created using average acoustic parameters derived from the speech of three male and three female adult speakers. The author used an F0-formantmatching technique in which, for some conditions, the acoustic parameters were consistent with the speaker gender, and at other times they were contradictory. The results revealed identification accuracy that was at $85 \%$ for appropriately matched female parameters and $97 \%$ for appropriately matched male parameters. In conditions with a male F0 (e.g., $120 \mathrm{~Hz}$ ) and female formant frequencies, identification as a male speaker was found at $94 \%$. For vowels containing a female F0 (e.g., $240 \mathrm{~Hz}$ ) and male formant frequencies, identification as a female was found at $75 \%$. These findings provided additional evidence supporting F0 as being an important acoustic cue for identifying speaker gender.

More recently, Gelfer and Mikos (2005) investigated gender identification from 250-ms vowel segments with various combinations of F0 and formant frequency values. The authors synthesized vowel signals based on acoustic analyses of F0 and F1-F3 for ten male, ten female, and ten male-tofemale transgendered speakers. The results revealed that listeners relied heavily upon F0 cues when identifying speaker gender. For example, when male formants were paired with a female appropriate F0, listeners identified the speaker as male $19 \%$ of the time, with listeners identifying the speaker as female $20 \%$ of the time when female formants were paired with male appropriate F0s. Poon and Ng (2011) offered similar evidence regarding the significant contribution of F0, but suggested that formants likely act as supplementary cues when F0 values fall within an ambiguous frequency range (i.e., 162-205 Hz). The authors synthesized two sets of vowels with various $\mathrm{F} 0$ and formant values using a vowel production by one male and one female speaker. Using Praat software, the first three formant values were multiplied by ten scale factors from 1 to 1.20 , and F0 was scaled to ten values between 100 and $250 \mathrm{~Hz}$ for the male vowels. For female vowels, the formant frequencies were multiplied by ten scale factors from 1 to 0.83 , with $\mathrm{F} 0$ values in the same frequency range as the male versions of the synthesized vowels. This allowed for the creation of signals comprised of male appropriate $\mathrm{F} 0$ and formant frequencies, female appropriate F0 and formant frequencies, and gender ambiguous F0 and formant frequencies. The results showed a significant influence of F0 value changes on identification but did not show a similar pattern when formant values were altered. More specifically, the likelihood of signals being perceived as male increased with decreasing F0, whereas the likelihood of signals being perceived as female increased with increased F0 values. For F0 values in the range of $162-205 \mathrm{~Hz}$, results showed significantly poorer identification of speaker gender. The authors suggested that when F0s fell within this frequency range, listeners may have utilized formant frequency information when unable to reliably make judgments regarding speaker gender from F0 cues. At high and low F0 values, however, the recognition of speaker gender was more robust. Additionally, Skuk and Schweinberger (2014) found F0 to be the most significant acoustic parameter (followed by formant frequencies, then spectrum level) for the gender identification of syllables produced by two male and two female speakers.

Hillenbrand and Clark (2009) performed pattern recognition using a quadratic discriminant analysis technique based on F0 and formant frequencies of 1,116 h-vowel-d (/hVd/) utterances produced by 45 men and 48 women from Hillenbrand, Getty, Clark, and Wheeler (1995). The results revealed that $\mathrm{F} 0$ values in isolation distinguished speaker gender slightly better (96\%) than normalized formant values (92\%) and the combination of these characteristics distinguished gender approximately as well as human listeners. On the basis of their findings, the authors suggested that both F0 and formant frequencies are required for robust gender identification, and that separate manipulations to each acoustic parameter were less effective at changing listener perception of speaker gender. Additionally, Gelfer and Bennett (2013) reported that the relative importance of F0 and formant frequencies differs depending on the type of stimulus used for experimental testing. Specifically, in brief speech signals (e.g., isolated vowels), F0 provides a more dominant cue for judging speaker gender, whereas in longer signals (e.g., connected speech), formant frequencies provide a stronger cue.

Taken together, these findings are somewhat equivocal regarding the relative contributions of F0 and low-frequency formant cues for determining speaker gender from an audio signal. What is apparent from the literature, however, is the preponderance of evidence documenting the importance of spectral information below F3 (i.e., approximately $3 \mathrm{kHz}$ ) for determining gender identity, with less known about the perceptual information contained within the high-frequency region of the speech spectrum (i.e., above F3). Given the scarcity of research in this area, it is important to study all spectral regions of the speech signal that may carry gender identity cues in order to develop a complete understanding of this perceptual process. This is particularly true given evidence documenting the benefits of extended spectral bandwidths for perceptual tasks including improvements in phoneme recognition and novel-word learning in children (e.g., Pittman, 2008; Stelmachowicz, Lewis, Choi, \& Hoover, 2007; Stelmachowicz, Pittman, Hoover, \& Lewis, 2001), speech recognition in children and adults (Mlot, Buss, \& Hall, 2010; Moore, Füllgrabe, \& Stone, 2010), computer segregation of speech in noise (Hu \& Wang, 2004), and individual 
speaker recognition by computer models in quiet (Hayakawa \& Itakura, 1994) and in noise (Deshpande \& Holambe, 2011; Hayakawa \& Itakura, 1995).

The purpose of this study was to evaluate the ability of normal-hearing listeners to identify speaker gender from high-pass filtered vowel segments produced by ten male and ten female adult speakers. The vowel signals used in this experiment were filtered to remove the traditionally studied acoustic information (i.e., information below approximately $3 \mathrm{kHz}$ ) for gender recognition. In other words, it was of interest to determine whether listeners could accurately identify speaker gender when the vowel segments were degraded through high-pass filtering. Given the documented importance of the low-frequency region for performing this perceptual task, the effects of removing low-frequency spectral detail are yet to be determined. If listeners are capable of performing the gender identification task, it would provide evidence of useful perceptual information regarding gender identity in the high-frequency region of the voiced speech signal.

\section{Experiment 1}

\section{Method}

Participants Thirty listeners (18 females, 12 males) with normal hearing between the ages of 18 and $35(M=22.7$ years, $S D=2.6$ years) participated in the experiment. Each listener was required to pass a hearing screening at $15 \mathrm{~dB} \mathrm{HL}$ for octave frequencies from 250 to $8000 \mathrm{~Hz}$ bilaterally. Highfrequency thresholds at $10,12.5,14$, and $16 \mathrm{kHz}$ were also screened at $15 \mathrm{~dB}$ HL bilaterally. Requiring listeners to pass the screening above the traditional $8 \mathrm{kHz}$ test frequency was done to ensure a minimum level of high-frequency sensitivity above $8 \mathrm{kHz}$, given that the stimuli contained only spectral energy above $3.5 \mathrm{kHz}$. None of the listeners were excluded from participation on the basis of this criterion.

Speakers Ten males and ten females between the ages of 18 35 served as speakers for the experiment. The speakers were negative for suspicion of hearing loss, negative for a history of voice therapy, and reported being in good health on the day of the recording session. Of the 20 speakers, $45 \%$ were from West Virginia, and the remainder were from the following states: Maryland (15\%), Pennsylvania (10\%), New York (10\%), Michigan (5\%), New Jersey (5\%), Virginia (5\%), and Florida (5\%). Because the experimental task required listeners to identify speaker gender rather than the individual vowel category, no effort was undertaken to control for speaker dialect. However, significant dialectical differences were not observed while recording the sustained vowels, which occurred immediately following a practice session. To control for the physical characteristics of the speakers, all were within two standard deviations for height and weight, according to the most recent data from the Centers for Disease Control Advance Data from Vital and Health Statistics (Ogden, Fryar, Carroll, \& Flegal, 2004). A two sample $t$-test found no significant difference in speaker age between the two genders, $t(9)=0.34, p=.73$.

Recording Recording took place in a sound-treated audio suite that met the ANSI S3.1-1999 (R2013), Maximum Permissible Ambient Noise Levels for Audiometric Test Rooms, standard for ears covered. The speakers were required to practice producing the vowels until they reported being comfortable and ready to begin the recording session. Each speaker produced the sustained vowel signals for approximately $1.5-2 \mathrm{~s}$ for multiple productions during the recording session. Recording the vowel signals in this manner allowed for the extraction of a steady-state portion of the vowel productions. A Miktek C1 FET (Nashville, TN) condenser microphone placed 12 in. from the speaker's mouth was used for recording. The speakers were instructed to maintain a constant speaking level and distance from the microphone while producing the vowels. The examiner observed the speakers during the entire recording session to ensure appropriate microphone-to-mouth distance. A sampling frequency of $44.1 \mathrm{kHz}$ with 24-bit amplitude resolution was used during the recordings.

Stimuli The stimuli for the experiment consisted of naturally produced, steady-state vowel segments of 250-ms duration.

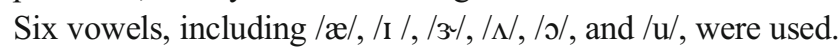
The vowel segments included two front, two central, and two back vowels to represent vowel productions from various locations in the vocal tract. A 250-ms steady-state segment from the center of the vowel segment was extracted from each of the six vowels. The vowel segments were then windowed using a 10-ms Hamming window to reduce onset/offset artifacts. Table 1 contains the average F0 and formant frequency information from the male and female vowel segments. These values were obtained using the Praat (Boersma \& Weenink, 2013) "Pitch" and "Show formant" commands for each vowel. The segments were then high-pass filtered at $3.5 \mathrm{kHz}$ (with a 100-Hz transition band) using a zero-phase Equiripple finiteimpulse response filter with $120-\mathrm{dB}$ attenuation in MATLAB (The Mathworks, Natick, MA). This filter cutoff was selected with the intent of removing important low-frequency acoustic detail, including F3 and below (see Table 1 for the highest F3 values, and Fig. 1 for the spectra of an unfiltered and a filtered vowel segment). As can be seen, spectral information below $3.5 \mathrm{kHz}$ was effectively removed from the vowel segment.

Procedure This study was approved by the West Virginia University Institutional Review Board for the Protection of Human Research Subjects. Listening sessions took place in 
Table 1 Mean fundamental and formant frequency information from male and female speakers, in hertz

\begin{tabular}{|c|c|c|c|c|c|c|c|}
\hline & & $/ æ /$ & $/ \mathrm{J} /$ & $\mid 3 w$ & $/ \mathrm{I} /$ & $\mid \Lambda /$ & $/ \mathrm{u} /$ \\
\hline \multirow[t]{2}{*}{ F0 } & M & 117 (12) & $121(16)$ & 124 (16) & $126(21)$ & $124(16)$ & $126(18)$ \\
\hline & $\mathrm{F}$ & $218(24)$ & 219 (19) & 221 (18) & $224(18)$ & $220(20)$ & $229(23)$ \\
\hline \multirow[t]{2}{*}{$\mathrm{F} 1$} & M & 714 (93) & 717 (96) & 599 (132) & 459 (67) & $656(104)$ & 414 (109) \\
\hline & $\mathrm{F}$ & 949 (106) & $809(60)$ & $622(61)$ & $546(59)$ & $699(61)$ & $424(50)$ \\
\hline \multirow[t]{2}{*}{$\mathrm{F} 2$} & M & 1667 (158) & $1068(95)$ & $1438(131)$ & 1909 (154) & $1312(203)$ & 1445 \\
\hline & $\mathrm{F}$ & $1920(131)$ & $1220(84)$ & $1650(135)$ & $2177(158)$ & $1530(110)$ & $1466(226)$ \\
\hline \multirow[t]{2}{*}{ F3 } & M & 2550 (164) & $2610(135)$ & $1907(326)$ & 2735 (157) & $2772(178)$ & $2538(307)$ \\
\hline & $\mathrm{F}$ & 3029 (190) & $3022(208)$ & $2149(332)$ & 3050 (193) & $2958(178)$ & $2859(228)$ \\
\hline
\end{tabular}

Standard deviation values in parentheses

a sound-treated audio suite in the Department of Communication Sciences and Disorders at West Virginia University. Gender identification abilities were evaluated using a two-alternative forced choice task. Each listener initially underwent a brief practice session to become familiarized with the graphical user interface used to collect responses, as well as with the filtered vowel segments. Prior to the practice session, the listener was instructed to make his or her selection by using a mouse to click on one of two rectangular buttons ("Male voice" or "Female voice") to indicate the gender of the speaker producing the sound. The listeners were randomly presented 16 unfiltered and 16 high-pass filtered vowel segments from two male and two female speakers who did not serve as speakers for the experimental tasks. The stimuli for the practice and experimental sessions were root-mean-square equalized in MATLAB (The Mathworks, Natick, MA). No feedback as to the accuracy of the participant's responses was provided.

Following the practice session, each listener was presented with six vowel segments from each of the ten male and ten female speakers (120 presentations) in an unfiltered condition and a high-pass filtered condition, for a total of 240 responses. Presentation order was counterbalanced to reduce order effects. Presentation of the individual segments was randomized during the experimental listening sessions. The vowel signals were presented diotically at $65 \mathrm{~dB}$ SPL via Sennheiser HD 380 Pro circumaural headphones (Old Lyme, CT). According to the manufacturer, these headphones have an extended frequency response to $27 \mathrm{kHz}$, and therefore ensured highfidelity presentation of the experimental stimuli. The listener was again instructed to click on the appropriate response box (i.e., "Male voice" or "Female voice") following presentation of each vowel segment. One second following the selection, a new vowel segment was randomly presented. All listeners completed the experimental tasks during one listening session lasting approximately $1 \mathrm{~h}$.

\section{Results}

Descriptive statistics A total of 7,200 responses (20 Talkers $\times$ 6 Vowels $\times 30$ Listeners $\times 2$ Stimulus Conditions) were elicited. The results were analyzed using the binomial test to
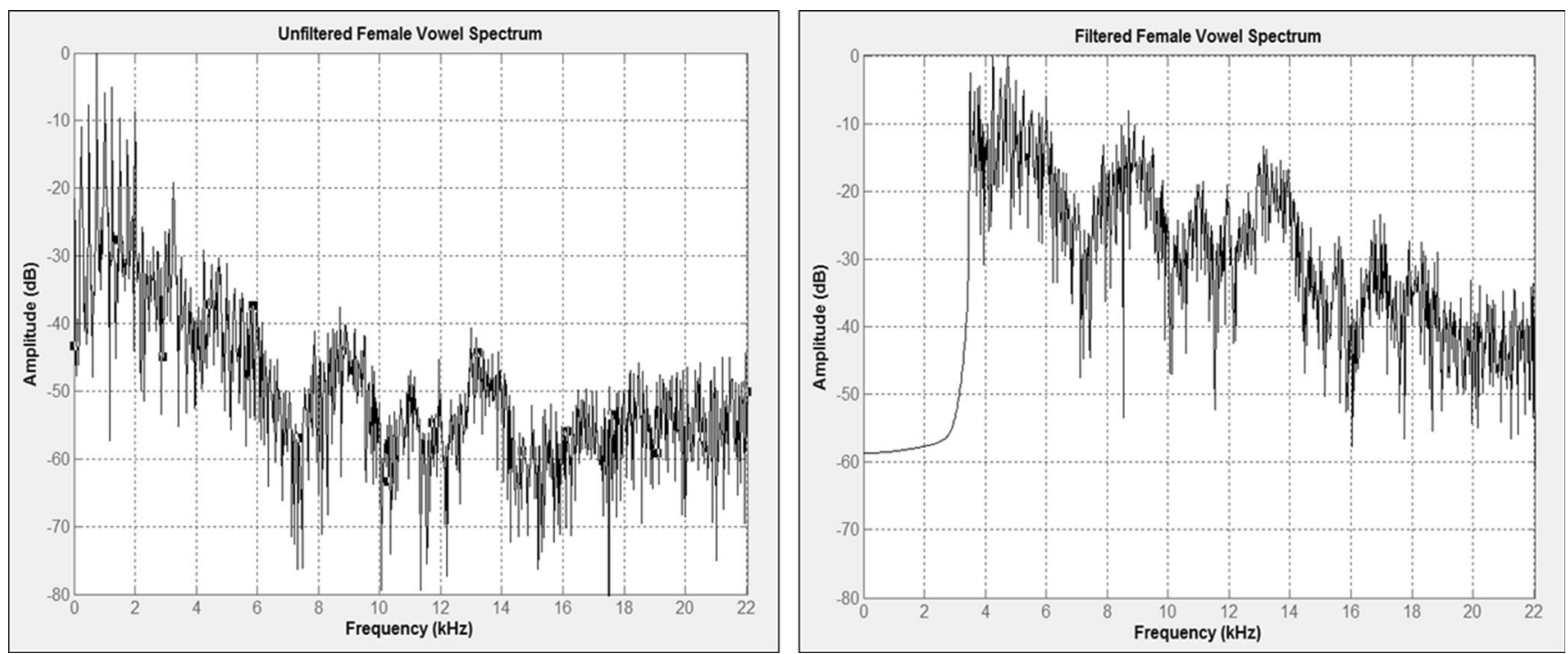

Fig. 1 Spectrum of unfiltered female segment (left) and filtered segment (right) 
determine whether identification performance was significantly above chance for each normal-hearing listener participating in the experiment. In order to reach performance significantly above chance using a two-tailed test $(p<.05)$, listeners must have correctly identified speaker gender for at least 72 of the 120 total vowel segments. To reach similar performance for the male and female segments individually, listeners must have correctly identified gender for 39 of the 60 segments.

The overall identification performance was found to be significantly above chance for all 30 listeners in both the unfiltered and filtered conditions. Further examination of the data revealed that each of the 30 listeners performed significantly above chance on the high-pass filtered male segments, whereas 28 performed similarly on the filtered female condition. Specifically, identification of the female high-pass filtered segments was observed to be significantly above chance for all but two listeners, whose scores were nonsignificant $(p>$ $.05)$ at $53 \%$ and $57 \%$, according to the binomial test. Therefore, for the remainder of the analyses, the data from these two listeners were removed.

The results from the unfiltered and filtered vowels are displayed in Table 2. As can be seen, gender identification was fairly uniform among the six unfiltered vowel segments, with increased variability noted among the filtered segments. Cumulative gender identification accuracy for the unfiltered vowel segments was found to be above $90 \%$ for each male and female segment, whereas identification of the filtered vowel segments ranged from $69 \%$ to $91 \%$. As is noted in Table 2, binomial test results (two-tailed) showed cumulative identification performance for each vowel category to be significantly above chance $(p<.05)$ for both the male and female speakers in the filtered listening condition. As expected, similar above-chance performance was also observed for the unfiltered vowel segments.

Table 3 displays the identification accuracy results from the vowel segments produced by each of the 20 speakers in the

Table 2 Identification accuracy for unfiltered and filtered vowel segments (percentages)

\begin{tabular}{llllll}
\hline & \multicolumn{2}{l}{ Unfiltered } & & \multicolumn{2}{l}{ Filtered } \\
\cline { 2 - 3 } \cline { 5 - 6 } Vowel & Male & Female & & Male & Female \\
\hline /æ/ & 99.33 & 98.21 & & $90.67^{*}$ & $73.21^{*}$ \\
$/ \mathrm{I} /$ & 98.21 & 93.93 & & $87.85^{*}$ & $78.33^{*}$ \\
/3/ & 98.57 & 96.43 & & $76.78^{*}$ & $87.51^{*}$ \\
/N/ & 99.29 & 93.93 & & $90.67^{*}$ & $79.67^{*}$ \\
/ $/$ & 98.57 & 96.07 & & $83.57^{*}$ & $79.29^{*}$ \\
/u/ & 100.00 & 96.43 & & $69.00^{*}$ & $90.71^{*}$ \\
Mean & 99 & 96 & & $83^{*}$ & $81^{*}$ \\
Overall & 97.5 & & $82^{*}$ & \\
\hline
\end{tabular}

${ }^{*}$ Indicates performance significantly above chance $(p<.05)$
Table 3 Identification accuracy for the ten male and ten female speakers (percentages)

\begin{tabular}{llllll}
\hline Male & Unfiltered & Filtered & Female & Unfiltered & Filtered \\
\hline $\mathrm{m} 1$ & 99 & 77 & $\mathrm{f} 1$ & 98 & 75 \\
$\mathrm{~m} 2$ & 100 & 93 & $\mathrm{f} 2$ & 99 & 91 \\
$\mathrm{~m} 3$ & 100 & 85 & $\mathrm{f} 3$ & 99 & 91 \\
$\mathrm{~m} 4$ & 100 & 79 & $\mathrm{f} 4$ & 95 & 81 \\
$\mathrm{~m} 5$ & 98 & 92 & $\mathrm{f} 5$ & 98 & 83 \\
$\mathrm{~m} 6$ & 100 & 74 & $\mathrm{f6}$ & 95 & 72 \\
$\mathrm{~m} 7$ & 96 & 74 & $\mathrm{f} 7$ & 97 & 71 \\
$\mathrm{~m} 8$ & 99 & 76 & $\mathrm{f} 8$ & 97 & 86 \\
$\mathrm{~m} 9$ & 97 & 92 & $\mathrm{f} 9$ & 81 & 72 \\
$\mathrm{~m} 10$ & 99 & 93 & $\mathrm{f} 10$ & 96 & 83 \\
\hline
\end{tabular}

unfiltered and filtered conditions. Overall, the gender identification performance for the ten male and ten female unfiltered productions was well above $90 \%$, with the exception of performance for one female speaker ( 99 ), at $81 \%$. The reason for the reduced identification from the unfiltered vowel segments produced by this individual is unknown but might have been a result of speaker dialect. However, as we previously described, the authors did not observe notable dialectal differences among the speakers during the recording session. Moreover, it should be noted that gender identification from the filtered vowel segments produced by this speaker was comparable to that from the other speakers in the experiment, with performance on the filtered vowel signals being of primary interest to the study.

Next, our results were analyzed to determine whether gender identification performance was significantly above chance for each of the ten male and ten female speakers from the experiment. Binomial test results revealed the overall identification performance to be above chance $(p<.05)$ for each male and female speaker in both the unfiltered and filtered conditions. Therefore, listeners in this experiment identified gender with performance significantly above chance from high-pass filtered vowel segments produced by the 20 individuals serving as speakers for the experiment. This indicates that speaker gender was identified well from the vowel segments produced by every speaker in the study.

Analysis of variance (ANOVA) Prior to the analysis, percent correct scores were transformed to rationalized arcsine units (Studebaker, 1985). A $2 \times 2 \times 6$ ANOVA was performed using Speaker Gender (male/female), Listening Condition (unfiltered/filtered), and Vowel Category (six vowels) as withinsubject factors. Where applicable, Mauchly's test was conducted and produced nonsignificant results $(p>.05)$, suggesting that the assumption of sphericity was met. Interpretation of the main effects and interactions most germane to the primary 
purpose of the study is provided. Pairwise comparisons were analyzed using Bonferroni corrections.

Significant main effects of speaker gender, $F(1,27)=7.18$, $p<.05$, and listening condition, $F(1,27)=180.31, p<.001$, were found, with a nonsignificant effect of vowel category, $F(5,135)=0.81, p=.54$. Further analysis revealed gender identification from the male segments to be significantly higher than that from the female segments, and that gender identification performance in the high-pass filtered condition was significantly lower. Moreover, the results revealed no significant differences in gender identification among the vowel categories. A significant interaction was found for speaker gender and vowel category, $F(5,135)=15.45$, $p<.001$, listening condition and vowel category, $F(5,135)=$ $2.81, p<.05$, and speaker gender, listening condition, and vowel category, $F(5,135)=15.49, p<.001$. A significant interaction was not observed, $F(1,27)=1.01, p=.32$, for speaker gender and listening condition, suggesting that the gender identifications from the male and female vowel segments were comparable in the unfiltered and filtered listening conditions. In other words, the deleterious effects of high-pass filtering were similar for both the male and female vowel segments.

\section{Supplementary Experiment 1 (S1): Gender identification in low-frequency shaped noise}

\section{Method}

Listeners in the previous experiment were capable of determining gender identity from high-pass filtered vowel segments with notable accuracy. To rule out the possibility that perceptual cues resulting from combination tones or other unintended low-frequency cues contributed to identification, ten additional listeners (seven females, three males) between the ages of 18 and 35 ( $M=22.5$ years, $S D=2.4$ years) completed an experimental session in the presence of lowfrequency noise masking. The use of ten normal-hearing listeners to confirm the results of Experiment 1 was comparable to the number of listeners (seven) from a recent study that confirmed gender identification and speech production mode judgments from speech filtered to contain only highfrequency information (see Monson, Lotto, \& Story, 2014, for details).

Combination tones can result from intermodulation distortion products when two or more sinusoidal components are presented simultaneously (Plack \& Oxenham, 2005). Therefore, a white-noise signal, which was low-pass filtered at $3.5 \mathrm{kHz}$ using a finite-impulse response filter with a $100-\mathrm{Hz}$ transition band and 120-dB attenuation, was presented simultaneously during the gender identification task, as we previously described. The masking signal was presented at $45 \mathrm{~dB}$ SPL (20 dB SPL below the presentation level of the filtered vowel segments) to mask, if present, unintended perceptual cues below the high-pass filter cutoff frequency, without creating an upward spread of masking of the high-frequency vowel signals. As in Experiment 1, the listeners underwent a practice session, without feedback as to the accuracy of their responses. Then, in a counterbalanced order, listeners participated in two listening sessions including the identification of the previously used high-pass filtered vowel signals both in quiet and in the presence of the masking noise previously described. The results were examined to determine whether the presence of the noise masker reduced gender identification performance, relative to the performance observed without masking noise.

\section{Results}

Mean gender identification accuracy was observed at $85 \%$ ( $S D=7.1$, range $70 \%-95 \%$ ) for the high-pass filtered vowels presented in quiet and $86 \%(S D=9.1$, range $71 \%-95 \%)$ when presented in masking noise. A paired sample $t$-test showed no significant difference in identification performance between the two listening conditions, $t(9)=0.92, p=.38$. Consequently, these findings confirmed the results of Experiment 1 in that the addition of masking noise below the high-pass filter cutoff frequency did not adversely influence listener ability to use high-frequency information to identify speaker gender. Moreover, identification performance for the ten additional listeners in quiet was comparable at $85 \%$ to that obtained in Experiment 1 (82\%), which further supports these initial findings.

\section{Supplementary Experiment 2 (S2): Gender identification from unequal signal proportions}

\section{Method}

To determine whether judgments made by listeners in Experiment 1 were truly associated with speaker gender identity from the high-pass filtered vowel segments, a second supplementary experiment was conducted. To assess this possibility, the stimulus sets for the experiment contained various proportions of male and female signals from Experiment 1. For example, one group of ten listeners (eight females, two males) between the ages of 18 and $35(M=23.8$ years, $S D=1.9$ years) made judgments from high-pass filtered vowel signals, using a set of high-pass filtered vowels containing $75 \%$ (60) male signals and $25 \%$ (20) female signals. The second group of ten listeners (eight females, two males) of similar age ( $M=23.2$ years, $S D=1.8$ years $)$ made judgments from $25 \%$ (20) male signals and $75 \%$ (60) female signals. The signals for the reduced proportions were randomly selected from the complete male or female stimulus set, whereas all 
of the original filtered signals were used for the stimulus set with the higher proportion. All listeners underwent a practice listening session, without feedback, as was previously described. Unlike in Experiment 1, the listeners in this supplementary task did not make gender identity judgments from the unfiltered vowel segments. All other methodological procedures, however, were identical.

\section{Results}

The overall identification accuracy for the stimulus set containing a greater proportion of male signals was found to be $88 \%(S D=5.1$, range $77 \%-97 \%)$, with $87 \%(S D=6.8$, range $75 \%-100 \%$ ) accuracy for the male signals and $90 \%$ $(S D=7.1$, range $80 \%-100 \%)$ accuracy for the female signals. The overall identification accuracy for the stimulus set containing a greater proportion of female signals was found to be $84 \%(S D=7.8$, range $74 \%-99 \%)$, with $91 \%(S D=5.7$, range $85 \%-100 \%$ ) accuracy for the male signals and $80 \%$ ( $S D=9.3$, range $70 \%-98 \%$ ) accuracy for the female signals. Thus, these results are similar to those observed in Experiment 1 , and consequently strengthen the perceptual judgments regarding gender identity elicited in the original experimental task. Additionally, the gender identification performance presented here concurs with the results reported by Monson et al. (2014), in which normal-hearing listeners identified speaker gender from speech (i.e., phrases from the "Star Spangled Banner") that contained only high-frequency energy (above approximately $5.7 \mathrm{kHz}$ ) with over $90 \%$ accuracy.

\section{Experiment 2}

\section{Discriminant analyses}

To explore potential cues for gender identity from the highpass filtered vowel segments, discriminant function analyses were performed to determine whether and how well the highpass filtered vowel segments could be separated using data from the gammatone filter bank excitation patterns, as well as information derived from autocorrelation functions (ACFs). The use of two signal representations (described below) was intended to evaluate classification performance by using a perceptually motivated spectral representation (basilar membrane excitation pattern) and a temporal representation (ACF) of the high-pass filtered vowel segments.

Linear discriminant analysis techniques were used for classification purposes. Cross-validation based on a leave-one-out technique was utilized to provide a more generalizable predictive model. In this technique, one of the test signals is removed from the dataset used for training the classifier (i.e., all but the signal to be classified are used as training data), then the signal originally removed is entered as a novel input and classified.
This cross-validation technique is reported to be suitable for providing accurate predictions for small datasets such as the one used in this experiment (Witten \& Frank, 2000).

\section{Classification using excitation pattern data}

Excitation peak information was derived from the highpass filtered segments using a gammatone filter bank implemented in MATLAB, with parameters from Glasberg and Moore (1990). The stimulus sample rate was $44.1 \mathrm{kHz}$ using 128 equivalent rectangular bandwidth (ERB) filters. An absolute threshold function to account for the differential frequency processing produced by the external and middle ears was applied using the Moore and Glasberg (1987) parameters. See Table 4 for the mean excitation pattern peak values derived from the 60 male and 60 female high-pass filtered segments and used in the classification, as well as Fig. 2 for an example excitation pattern. Two individuals, including the first author and an individual external to the study, visually examined the patterns plotted in MATLAB to determine the most prominent excitation peaks. A third individual reviewed $20 \%$ of the excitation patterns, which resulted in $100 \%$ agreement for the values selected by the first two evaluators. Upon completion, it was determined that the data from the first three prominent peaks would be used in the analysis, because all filtered segments created an excitation pattern containing at least three prominent peaks, whereas only a small number of segments created excitation patterns containing four peaks.

Discriminant function analysis results suggested that the peak values from excitation patterns of the high-pass filtered vowel segments were significant predictors of speaker gender $\left(\chi^{2}=50.7, d f=3, p<.01\right)$ and accounted for $35.3 \%$ of the variance in predicting gender identity. Classification results from the peak excitation values revealed $80 \%$ correct classifications of speaker gender from the high-pass filtered vowel segments. As can be seen in the perceptual data, fewer classification errors were found for the filtered male segments $(10 \%)$ than for the female segments $(30 \%)$. Specifically, 54 of the 60 male segments were classified as male, with 42 of 60 segments being correctly classified as female.

Table 4 Mean equivalent rectangular bandwidth (ERB) frequency values from male and female vowel segments

\begin{tabular}{llll}
\hline & Peak 1 & Peak 2 & Peak 3 \\
\hline Male & $80(1.5)$ & $93(4.5)$ & $103(5.1)$ \\
Female & $83(2.1)$ & $96(5.7)$ & $107(5.2)$ \\
\hline
\end{tabular}

Standard deviations in parentheses 


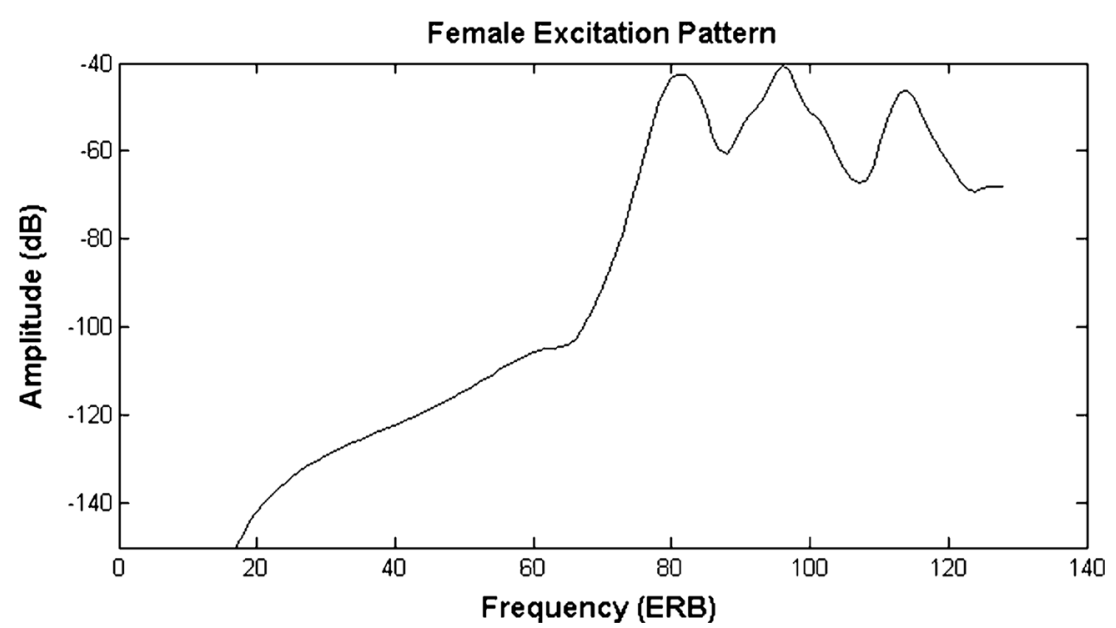

Fig. 2 Example excitation pattern derived from a high-pass filtered female vowel segment

\section{Classification using ACF data}

ACFs represent the sum of the multiplications of a signal by a shifted version of itself and are customarily used to derive the periodicity of a signal (von dem Knesebeck \& Zölzer, 2010). ACFs display peaks at time intervals (i.e., time lags) with high correlation, with the highest peak following the zero time lag representing the periodicity of the signal, or lack thereof. For the purposes of this experiment, ACFs were obtained using Praat's "Autocorrelate" function, found within the "Analyse Periodicity" analysis section. Normalized amplitude scaling was used for the analysis. ACF values were computed for the 60 male and 60 female high-pass filtered vowel segments used in the experiment. The values from the first five prominent peaks (time lags) in the ACF were used in the discriminant analysis. Examples created in Praat for one male and one female high-pass filtered segment are provided in Fig. 3. Two individuals, including the first author and another individual external to the study, were involved in selecting peak values from the ACFs of the high-pass filtered vowel segments. A third individual reviewed $20 \%$ of the ACF values, which resulted in $92 \%$ agreement for the values selected by the first two evaluators.
The discriminant function analysis results suggested that time lag information from ACFs derived from the high-pass filtered vowel segments was a significant predictor of speaker gender $\left(\chi^{2}=92.7, d f=5, p<.01\right)$ and accounted for $55.2 \%$ of the variance in predicting gender identity. The classification results using ACF lag values revealed $88 \%$ correct classifications of speaker gender from the high-pass filtered vowel segments. Again, as we observed in the perceptual data, fewer classification errors were found for the male segments $(8 \%)$ than for the female segments $(15 \%)$. For this signal representation, 55 of the 60 male segments were correctly classified as male, and 51 of 60 segments were correctly classified as female.

\section{Discussion}

The results of this experiment revealed that normal-hearing listeners were capable of extracting perceptual information regarding gender identity from naturally produced, high-pass filtered vowel segments. The stimuli in this experiment were filtered to remove spectral information below $3.5 \mathrm{kHz}$ (i.e., F0 and F1-F3), the frequency region commonly studied for its
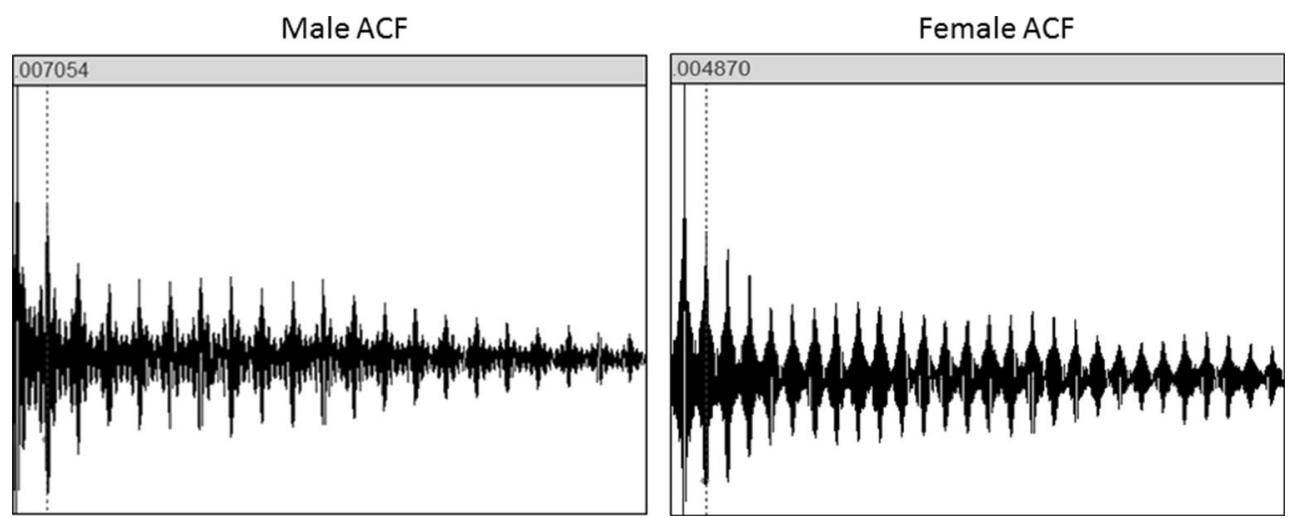

Fig. 3 Autocorrelation functions (ACFs) derived from one each of a high-pass filtered male (left) and female (right) segment 
contributions to gender identity (Gelfer \& Bennett, 2013; Gelfer \& Mikos, 2005; Lass, Hughes, Bowyer, Waters, \& Bourne, 1976; Poon \& Ng, 2011; Skuk \& Schweinberger, 2014; Whiteside, 1998). Considering evidence documenting the utility of additional high-frequency information for speech perception and novel-word learning in children (Pittman, 2008; Stelmachowicz et al., 2007; Stelmachowicz et al., 2001) and the automatic recognition of speech and/or speaker by computers (Deshpande \& Holambe, 2011; Hayakawa \& Itakura, 1994, 1995; Hu \& Wang, 2004), we hypothesized that listeners would perform above chance on the experimental gender identification task, due to the presence of useful perceptual information in the high-frequency region of the speech spectrum. This hypothesis was confirmed. Despite the removal of low-frequency spectral energy from the vowel segments, gender identification was significantly above chance for nearly every listener participating in the experiment, with chance representing the predicted level of performance following the removal of salient low-frequency acoustic detail. This was also the case in the presence of the low-frequency masker utilized in Supplementary Experiment 1, which was designed to confirm that listeners were not relying on unforeseen lowfrequency cues. Supplementary Experiment 2 was designed to assess the validity of the gender identification task from Experiment 1 using variable proportions of the male and female filtered segments. These identification results were comparable to those from the original experiment, and therefore strengthen the perceptual findings.

Together, these results coincide with recent data reported by Monson et al. (2014). These authors examined gender and mode of production determination from spoken and sung speech filtered to only contain information above approximately $5.7 \mathrm{kHz}$. Listeners in their experiment were capable of extracting perceptual information regarding speaker gender and mode of production using passages from the "Star Spangled Banner." The authors reported gender identification scores above $90 \%$ for both spoken and sung productions. Although gender identification from their study was higher than that reported in the present study, this is likely explained by the fact that the more complex stimuli (i.e., phrases) used in the Monson et al. study could have contained cues in addition to the vowel segments of equal duration used in the present experiment. Irrespective of these differences, both studies confirm the presence of perceptual cues in the high-frequency region of the speech spectrum.

For some listeners from the present study, the removal of low-frequency spectral energy decreased identification performance by approximately $5 \%-10 \%$, whereas for other listeners identification accuracy was reduced by $25 \%-30 \%$. This suggests the deleterious effects of high-pass filtering to be more substantial for some normal-hearing listeners than for others. In other words, some listeners were more adept at identifying speaker gender from the high-pass filtered vowel segments than others, despite being matched for hearing sensitivity. This degree of variability was also observed in a previous study examining vowel category judgments from highpass filtered male, female, and child vowels (Donai \& Paschall, 2015). The normal-hearing listeners in Donai and Paschall's study showed substantial differences in their abilities to identify vowel category from naturally produced, highpass filtered $/ \mathrm{hVd} /$ tokens from two adult male, two adult female, and two child speakers. The reason for the observed variability in using acoustic information from the highfrequency region for perceptual tasks in both studies has yet to be determined. Nevertheless, the listeners in the present experiments proved to be exceptionally accurate when judging speaker gender from naturally produced, high-pass filtered vowel signals. In light of the findings reported by Monson et al. (2014), Donai and Paschall, and the present authors, it is plausible to suggest the existence of useful perceptual information in the high-frequency region of naturally produced speech (i.e., above F3).

\section{Computer recognition of speech}

Previous research has documented the benefits of highfrequency information for both individual speaker recognition and separating speech from noise in a degraded acoustic signal. For example, Hayakawa and Itakura (1994) investigated the contributions of acoustic features extracted from specified bandwidths on automated speaker recognition. The results revealed similar recognition performance using features from the 0 - to $4-\mathrm{kHz}$ bandwidth as when using features from the 4- to $10-\mathrm{kHz}$ region, suggesting that the higher frequency region contains comparable amounts of information for the automatic recognition of individual speakers. Moreover, speaker recognition was highest at $96.4 \%$ in the widest bandwidth (i.e., $0-16 \mathrm{kHz}$ ). In a related study, Hayakawa and Itakura (1995) examined the effects of bandwidth on individual speaker recognition in various noise sources. The results revealed the benefits of using information from a $10 \mathrm{kHz}$ bandwidth in the process of automated speaker recognition in noisy environments. Furthermore, the authors suggested the 4- to $10-\mathrm{kHz}$ frequency band to be "more robust to noise" (p. 324) than lower-frequency bandwidths. Additionally, $\mathrm{Hu}$ and Wang (2004) designed an algorithm to segregate speech from a speech in noise mixture. The authors processed harmonic information from the low-frequency region (i.e., resolved harmonics) differently than harmonic information from the high-frequency region (i.e., unresolved harmonics). Specifically, the authors reported that unresolved harmonics contain strong amplitude modulations and the signal envelope fluctuates at the F0 of the target speech. The results revealed that the differential processing scheme, including additional high-frequency components, improved speech segregation performance in noise by a minimum of 
$3 \mathrm{~dB}$ signal-to-noise ratio (5.2- $\mathrm{dB}$ average improvement), as compared to previous versions lacking information from the high-frequency portion of the spectrum. More recently, Deshpande and Holambe (2011) investigated individual speaker recognition from sentences in the presence of competing car noise. Similar to previous studies, the authors reported significant information regarding individual speakers to exist in the high-frequency band above $4 \mathrm{kHz}$. Specifically, speaker identification using features from the 4- to $8-\mathrm{kHz}$ region resulted in $94.66 \%$ accuracy, again suggesting the high-frequency band to contain useful features for speaker recognition in ecological car noise. Taken together, these findings, in addition to the present results, support the supposition that useful perceptual information exists within the high-frequency region of the speech signal.

Consequently, it appears practical at this point to offer some possible explanations for these findings. Specifically, what acoustic cue(s) might have been available for determining gender identity in the high-frequency region of naturally produced vowel segments? Discriminant function analyses using both a spectral (excitation pattern) and a temporal (ACF) representation revealed classification of speaker gender approximating that from the perceptual data. Therefore, it is reasonable at this point to consider both spectral and temporal signal properties as possible explanations for the noteworthy gender identification performance observed in this study.

\section{Gender identification from spectrally degraded speech}

Evidence by Schvartz and Chatterjee (2012) has shown the gender identification abilities of normal-hearing listeners to be resistant to spectral degradation. In their experiment, the authors varied the amounts of spectral and temporal information available to listeners during a gender identification task. The results revealed that young normal-hearing listeners were capable of utilizing temporal information (i.e., temporal envelope cues) to establish speaker gender when spectral detail was degraded through noise-vocoding. However, the spectral degradation in the present experiment differed from that described in Schvartz and Chatterjee, since the listeners in our experiment were required to make gender identity judgments from vowel segments spectrally degraded via high-pass filtering. Given the limited frequency-resolving properties of the peripheral auditory system for high-frequency signals (Bernstein \& Oxenham, 2003; Dai, 2000; Micheyl, Keebler, \& Oxenham, 2010; Yost, 2009), it is conceivable that listeners in the present experiment relied on some form of temporal cues (e.g., residual temporal envelope or temporal fine structure) to identify speaker gender. Bernstein and Oxenham (2003) reported that when only harmonics above the 10th are present (as was the case in the present experiment), the auditory system utilizes temporal envelope cues in extracting pitch, but these percepts of pitch are often ambiguous and can lead to reduced F0 discrimination. Thus, it is possible that the listeners in this experiment were extracting periodicity information (as demonstrated using the ACF data) from the temporal envelope of the high-pass filtered vowel signals when identifying speaker gender. The precise mechanisms, however, are yet to be determined and are beyond the scope of this study, which was intended to obtain initial perceptual and classification data. Research to determine the contributions of spectral and temporal cues for identifying speaker gender from high-pass filtered vowel segments is underway.

\section{Conclusion}

The results of this experiment indicate that despite the removal of important low-frequency spectral detail, normal-hearing listeners were able to identify speaker gender with substantial accuracy. On the basis of these data, it is reasonable to suggest the presence of useful perceptual information related to gender identity in the high-frequency region of naturally produced vowel signals. Utilizing stimuli of equivalent duration eliminated perceptual cues such as the syllable duration and/or intonational patterns associated with signals of increased length and complexity (e.g., $/ \mathrm{hVd} / \mathrm{s}$, words, sentences, etc.), such as those used in Monson et al. (2014). Given that listeners were able to accurately identify speaker gender from vowel segments of equal duration, it is probable that more ecological speech signals (i.e., natural speech discourse) contain additional perceptual cues related to gender identity.

These results should not be interpreted as suggesting lowfrequency spectral information to be unimportant for identifying speaker gender. Rather, they provide evidence supporting the study of perceptual information for gender identity in the high-frequency region of the speech spectrum. Future research should investigate the type of information (e.g., spectral and/ or temporal) available in the high-frequency portion of the speech signal and the perceptual mechanisms used by the listeners in the present experiment. The goal of such investigations should be to elucidate the perceptual information in the high-frequency region of natural speech that is available for gender identification. This information has implications for the robust recognition of speech, and speaker gender in particular, in noisy and/or degraded acoustic environments.

As we previously noted, Hayakawa and Itakura (1994, 1995) demonstrated the benefits of including additional high-frequency speech information in individual speaker recognition systems. Specifically, the authors found that extending the spectral bandwidth of the speech signal significantly improved speaker recognition performance. In acoustic environments with considerable levels of low-frequency spectral energy (e.g., a busy office, hospital lobby, road traffic, and computer room), the authors found the addition of high- 
frequency speech energy to be advantageous for individual speaker recognition purposes. Additionally, similar results were found in the presence of car noise (Deshpande \& Holambe, 2011). As such, it is probable that in degraded acoustic environments (e.g., those containing substantial low-frequency energy), gender cues found in the highfrequency region of speech may be less susceptible to the deleterious effects of environmental noise sources and may provide valuable information to listeners. Future research should explore the perceptual nature of this understudied frequency region, with the goal of developing a more comprehensive understanding of the contributions of the acoustic information contained therein.

\section{References}

Bernstein, J. G., \& Oxenham, A. J. (2003). Pitch discrimination of diotic and dichotic tone complexes: Harmonic resolvability or harmonic number? Journal of the Acoustical Society of America, 113, 33233334.

Boersma, P., \& Weenink, D. (2013). Praat: Doing phonetics by computer (Version 5.3.56) [Computer program]. Retrieved November 2013 from www.praat.org/

Dai, H. (2000). On the relative influence of individual harmonics on pitch judgment. Journal of the Acoustical Society of America, 107, 953959

Deshpande, M. S., \& Holambe, R. S. (2011). Robust speaker identification in the presence of car noise. International Journal of Biometrics, 3, 189-205.

Donai, J. J., \& Paschall, D. D. (2015). Identification of high-pass filtered male, female, and child vowels: The use of high-frequency cues. Journal of the Acoustical Society of America, 137, 1971-1982.

Gelfer, M. P., \& Bennett, Q. E. (2013). Speaking fundamental frequency and vowel formant frequencies: Effects on perception of gender. Journal of Voice, 27, 556-566.

Gelfer, M. P., \& Mikos, V. A. (2005). The relative contributions of speaking fundamental frequency and formant frequencies to gender identification based on isolated vowels. Journal of Voice, 19, 544-554.

Glasberg, B. R., \& Moore, B. C. J. (1990). Derivation of auditory filter shapes from notched-noise data. Hearing Research, 47, 103-138.

Hayakawa, S., \& Itakura, F. (1994). Text dependent speaker recognition using the information in the higher frequency band. In Proceedings of the IEEE International Conference on Acoustics, Speech and Signal Processing (pp. 137-141). Piscataway, NJ: IEEE Press.

Hayakawa, S., \& Itakura, F. (1995). The influence of noise on the speaker recognition performance using the higher frequency band. In Proceedings of the IEEE International Conference on Acoustics, Speech and Signal Processing (pp. 321-324). Piscataway, NJ: IEEE Press.

Hillenbrand, J. M., \& Clark, M. J. (2009). The role of $f_{0}$ and formant frequencies in distinguishing the voices of men and women. Attention, Perception, \& Psychophysics, 71, 1150-1166. doi:10. 3758/APP.71.5.1150

Hillenbrand, J. M., Getty, L. A., Clark, M. J., \& Wheeler, K. (1995). Acoustic characteristics of American English vowels. Journal of the Acoustical Society of America, 97, 3099-3111.

Hu, G., \& Wang, D. (2004). Monaural speech segregation based on pitch tracking and amplitude modulation. IEEE Transactions on Neural Networks, 15, 1135-1150. doi:10.1109/TNN.2004.832812
Lass, N. J., Hughes, K. R., Bowyer, M. D., Waters, L. T., \& Bourne, V. T. (1976). Speaker sex identification from voiced, whispered, and filtered isolated vowels. Journal of the Acoustical Society of America, 59, 675-678.

Micheyl, C., Keebler, M. V., \& Oxenham, A. J. (2010). Pitch perception for mixtures of spectrally overlapping harmonic complex tones. Journal of the Acoustical of America, 128, 257-269.

Mlot, S., Buss, E., \& Hall, J. W. (2010). Spectral integration and bandwidth effects on speech recognition in school-aged children and adults. Ear \& Hearing, 31, 56-62.

Monson, B. B., Lotto, A. J., \& Story, B. H. (2014). Gender and vocal production mode discrimination using the high frequencies for speech and singing. Frontiers in Psychology, 5(1239), 1-7. doi:10. 3389/fpsyg.2014.01239

Moore, B. C. J., Füllgrabe, C., \& Stone, M. A. (2010). Effect of spatial separation, extended bandwidth, and compression speed on intelligibility in a competing-speech task. Journal of the Acoustical Society of America, 128, 420-433.

Moore, B. C. J., \& Glasberg, B. R. (1987). Formulae describing frequency selectivity as a function of frequency and level, and their use in calculating excitation patterns. Hearing Research, 28, 209-225.

Ogden, C. L., Fryar, C. D., Carroll, M. D., \& Flegal, K. M. (2004). Mean body weight, height, and body mass index, United States 1960-2002 (Advance Data From Vital Health Statistics, No. 347). Hyattsville, MD: National Center for Health Statistics.

Pittman, A. L. (2008). Short-term word learning rate in children with normal hearing and children with hearing loss in limited and extended high-frequency bandwidths. Journal of Speech, Language, and Hearing Research, 51, 785-797.

Plack, C. J., \& Oxenham, A. J. (2005). The psychophysics of pitch. In C. Plack, A. Oxenham, R. Fay, \& A. Popper (Eds.), Pitch: Neural coding and perception (pp. 7-55). New York, NY: Springer.

Poon, S.F., \& Ng, M. L. (2011). Contribution of voice fundamental frequency and formants to the identification of speaker's gender. In Proceedings of the 17th International Congress of Phonetic Sciences (pp. 1630-1633). Victoria, BC: International Phonetic Association.

Schvartz, K. C., \& Chatterjee, M. (2012). Gender identification in younger and older adults: Use of spectral and temporal cues in noisevocoded speech. Ear \& Hearing, 33, 411-420.

Skuk, V. G., \& Schweinberger, S. R. (2014). Influences of fundamental frequency, formant frequencies, aperiodicity, and spectrum level on the perception of voice gender. Journal of Speech, Language, and Hearing Research, 57, 285-296.

Stelmachowicz, P. G., Lewis, D. E., Choi, S., \& Hoover, M. A. (2007). The effect of stimulus bandwidth on auditory skills in normalhearing and hearing-impaired children. Ear \& Hearing, 28, 483494.

Stelmachowicz, P. G., Pittman, A. L., Hoover, B. M., \& Lewis, D. E. (2001). Effect of stimulus bandwidth on the perception of $/ \mathrm{s} /$ in normal-and hearing-impaired children and adults. Journal of the Acoustical Society of America, 110, 2183-2190.

Studebaker, G. A. (1985). A rationalized arcsine transform. Journal of Speech and Hearing Research, 28, 455-462.

von dem Knesebeck, A., \& Zölzer, U. (2010, September). Comparison of pitch trackers for real-time guitar effects. Proceedings of the 13th International Conference of Digital Audio Effects. Austria, September 6-10.

Whiteside, S. P. (1998). The identification of speaker's sex from synthesized vowels. Perceptual and Motor Skills, 87, 595-600.

Witten, I. H., \& Frank, E. (2000). Credibility: Evaluating what has been learned. In I. H. Witten \& E. Frank (Eds.), Data mining: Practical machine learning tools and techniques with Java implementations (pp. 119-156). San Diego, CA: Academic Press.

Yost, W. A. (2009). Pitch perception. Attention, Perception, \& Psychophysics, 71, 1701-1715. doi:10.3758/APP.71.8.1701 